\title{
Enzyme Replacement Therapy Stabilized White Matter Lesion Progression in Fabry Disease
}

\author{
Andreas Fellgiebel $^{a} \quad$ Martin Gartenschläger $^{b} \quad$ Kerstin Wildberger $^{a}$ \\ Armin Scheurich $^{a}$ Robert J. Desnick ${ }^{c}$ Katherine Sims ${ }^{d}$ \\ Departments of a Psychiatry and Psychotherapy, ${ }^{\mathrm{b}}$ Diagnostic and Interventional Radiology, University Medical \\ Center Mainz, Mainz, Germany; ${ }^{C}$ Department of Genetics and Genomic Sciences Mount Sinai School of \\ Medicine, New York, N.Y., d Department of Neurology, Massachusetts General Hospital, Harvard Medical School, \\ Boston, Mass., USA
}

\section{Key Words}

Fabry disease - White matter lesions - Enzyme replacement therapy

\begin{abstract}
Background: The central nervous system manifestations in Fabry disease (FD) include progressive white matter lesions (WMLs) and stroke. Due to progressive microvascular involvement, men and women with FD over 35 years of age develop WMLs. Moreover, the prevalence of stroke has been estimated to be 12 times higher in FD compared with the general population. Enzyme replacement therapy (ERT) is available and has shown beneficial effects on renal, cardiac, and peripheral nerve function in FD, but the ERT effect on the progression of WMLs, or the reduction in cerebrovascular events, remains unknown. Methods: The WML burden and the effect of agalsidase beta $1 \mathrm{mg} / \mathrm{kg}$ biweekly on WML progression were assessed longitudinally in a Phase 4 agalsidase-beta placebo-controlled analysis of untreated and treated FD patients with mild-to-moderate renal involvement (serum creatinine measurements of $\geq 1.2 \mathrm{mg} / \mathrm{dl}$ and
\end{abstract}

$<3.0 \mathrm{mg} / \mathrm{dl}$ ). The primary end point was the difference in the number of patients with increased WML burden between the agalsidase beta and placebo groups at the end of treatment. The diameters of the WMLs were determined manually using axial flow-attenuated-inversion-recovery-weighted magnetic resonance imaging (MRI) scans taken at baseline and follow-up. Results: MRI scans from 41 FD patients (mean age 43.9, age range 20-68, 3 females; $n=25$ on ERT, $\mathrm{n}=16$ on placebo) were analyzed. WML burden was present in $63 \%$ of patients at baseline, increased over a mean of 27 months (range 12-33 months) follow-up, and correlated with left ventricular hypertrophy (LVPW). Patients with previous or recent strokes ( $n=11,39-68$ years) showed an increase in the number of WMLs $(p=0.005)$. A greater proportion of younger patients ( $\leq 50$ years) on ERT $(n=18)$ had stable WML burden compared with younger patients in the placebo group ( $n=13$ ): $44 \%$ ( 8 of 18 ) versus $31 \%$ ( 4 of 13 ), $p=0.014$. The number needed to treat was 8 . Conclusions: This FD patient cohort, with mild-to-moderate renal involvement, had a significant WML burden and high inter-individual variability associated with the degree of LVPW but not the degree of kidney dysfunction. These advanced patients

\begin{tabular}{ll}
\hline KARGER & $\begin{array}{l}\text { ( 2014 S. Karger AG, Basel } \\
\text { 1015-9770/14/0386-0448 } \$ 39.50 / 0 \quad \text { Karger }\end{array}$ \\
$\begin{array}{l}\text { E-Mail karger@karger.com } \\
\text { www.karger.com/ced }\end{array}$ & $\begin{array}{l}\text { This is an Open Access article licensed under the terms of the } \\
\text { Creative Commons Attribution-NonCommercial 3.0 Un- } \\
\text { ported license (CC BY-NC) (www.karger.com/OA-license), } \\
\text { applicable to the online version of the article only. Distribu- } \\
\text { tion permitted for non-commercial purposes only. }\end{array}$
\end{tabular}

Prof. Dr. Andreas Fellgiebel, MD

Department of Psychiatry and Psychotherapy

University Medical Center Mainz

Untere Zahlbacher Strasse 8, DE-55131 Mainz (Germany)

E-Mail andreas.fellgiebel@unimedizin-mainz.de 
with increased LVPW and stroke evidence may have had a higher cerebrovascular risk. The WML burden in patients on ERT was more likely to remain stable, compared with patients on placebo. Thus, ERT may reduce the progression of vascular disease, even in advanced FD patients, suggesting that early treatment may stabilize WML progression and stroke risk.

(c) 2014 S. Karger AG, Basel

\section{Introduction}

Fabry disease (FD) is an X-linked lysosomal storage disease resulting from the deficient activity of alpha-galactosidase A ( $\alpha-\mathrm{Gal} \mathrm{A})$. In classically affected patients with essentially no detectable $\alpha-G a l$ A activity, the progressive accumulation of globotriaosylceramide (Gb3) in microvascular endothelial and smooth muscle cells results in microvascular ischemia and infarction leading to renal and neurologic manifestations including renal failure, transient ischemic attacks (TIAs), and stroke [1]. Double-blind, placebo-controlled multicenter Phase 3 and 4 clinical trials have shown that enzyme replacement therapy (ERT) with agalsidase beta clears the accumulated glycosphingolipid in the vascular endothelial cells of the kidney, heart, and skin [2], and slows the progression of combined renal, cardiac, and cerebrovascular events in patients with advanced FD [3]. However, central nervous system (CNS) symptoms remain a major burden in both untreated and treated patients, particularly in those with advanced FD, such that the prevalence of stroke has been estimated to be 12 times higher in patients with FD compared with the general population [4]. A major magnetic resonance imaging (MRI) finding in men and women with FD over 35 years of age is the presence of white matter lesions (WMLs) in the CNS [5], which result from progressive microvascular involvement. Microvascular disease is predominantly clinically asymptomatic; thus, brain imaging can be used to detect small cerebral infarcts (lacunae), before they manifest clinically, and provide information about the progression of disease.

WMLs are generally regarded as risk factors for stroke, cognitive decline, dementia, and death [6-8]. Although ERT in FD has been shown to reverse increased cerebral blood flow [9], the effect of ERT on the progression of WMLs or the risk of consecutive cerebrovascular events remains unknown [10]. Thus, this analysis was conducted to evaluate the effect of agalsidase beta on WML burden in patients with advanced FD.

\section{Methods}

This was a post hoc analysis of data from patients enrolled in a Phase 4 study of the safety and efficacy of agalsidase beta in patients with FD (ClinicalTrials.gov Identifier: NCT00074984) [3]. Institutional review boards at all sites approved the protocol. All patients gave written informed consent. Eighty-two adult patients ( 72 men, 10 women) with FD and evidence of kidney disease (serum creatinine measurements of $\geq 1.2 \mathrm{mg} / \mathrm{dl}$ and $<3.0 \mathrm{mg} / \mathrm{dl}$ ) were randomized to agalsidase beta $1 \mathrm{mg} / \mathrm{kg}$ biweekly or placebo. All patients had $\alpha$-Gal A activity $<1.5 \mathrm{nmol} / \mathrm{h}$ per $\mathrm{ml}$ of plasma (normal mean \pm standard deviation [SD] $8.1 \pm 3.2 \mathrm{nmol} / \mathrm{h}$ per $\mathrm{ml}$ of plasma) or $<4 \mathrm{nmol} / \mathrm{h}$ per $\mathrm{mg}$ of leukocyte protein (normal mean, $97.5 \pm$ $16.4 \mathrm{nmol} / \mathrm{h}$ per $\mathrm{mg}$ of leukocyte protein).

The presence or absence of the following cerebrovascular risk factors was obtained from the study participant's medical record: previous stroke, previous TIA, arterial hypertension, diabetes mellitus, atrial fibrillation, hyperlipoproteinemia, and smoking. Head MRI was used to assess WML progression and incidence of silent cerebral infarctions, by comparing scans performed at baseline and after at least 12 months of follow-up according to the methods outlined below.

\section{MRI Imaging Protocol}

Axial T1- (TR/TE: $475 \mathrm{~ms} / 12-16 \mathrm{~ms}$, Matrix $256 \times 192$, slice thickness $5 \mathrm{~mm}$, no gap, FoV $220 \times 220$ ) and axial flow-attenuatedinversion-recovery (FLAIR)-weighted (TR/TE 11,000 ms/144 ms, slice thickness $3 \mathrm{~mm}$, no gap, Matrix $256 \times 192$, FoV $220 \times 220$ ) images were used for analyses of WMLs, pulvinar signs, and stroke identification and classification. Hard copies (films) of longitudinal cranial MRI scans were collected for examination.

To ensure consistency, all MRI examinations were conducted under the same strict conditions. A patient's initial scan was performed within 28 days of study enrollment, and then every 3 months during the treatment period, with a final scan taken within 28 days (if possible) of the end of participation (defined as withdrawal or occurrence of a clinically significant renal [33\% increase in serum creatinine, dialysis or transplant], cardiac [myocardial infarction, significant change in cardiac status, that is, angina, congestive heart failure, or symptomatic arrhythmia requiring medication or surgery] or cerebrovascular [stroke or TIA] event and/or death [from any cause]). At each site, examinations were performed serially on the same MR machine (superconducting MRI system producing 1.0 Tesla or equivalent quality images) under the same scanning conditions for all patients, including axial or oblique orientation as needed and position and angle of slicing (along the orbital-meatal line with the imaging area extending from the vertex to the base of the skull).

\section{WML Measurements}

WMLs were defined as hyperintense signal abnormalities surrounding the ventricles and in the deep white matter on FLAIRand T2-weighted images, which did not have a hypointense center [11]. Lesion diameters were measured manually using a modified method that was previously described by Fellgiebel et al. [12]. Lesion-diameter measurements were normalized using the sagittal anterior-posterior skull diameter for each individual as an internal standard to compensate for any differences in the magnification factor resulting from the use of printed films. Areas classified as 
stroke by our neuroradiologist (M.G.) were excluded from the analysis, but no lower/upper limit restrictions were applied to the WML diameter.

\section{Left-Ventricular Posterior Wall Measurements}

End-diastolic thickness of the LVPW was determined using standard 2D targeted M-mode echocardiogram recordings according to American Society of Echocardiography methods.

\section{Stroke Identification and Classification}

Cerebral infarctions were identified as present or absent on each brain image. Each infarction was recorded by vascular territory and type (small-vessel lacunae or large-vessel territory).

\section{Reliability Analyses}

The reliability of the analyses was assessed by four trained raters. After evaluation of imaging quality and removal of scans showing severe artifacts, inter-rater reliability was determined. Each single rater independently analyzed the same 12 randomly selected test scans. After achieving a good inter-observer reliability (intra-class correlation mean 0.977; 95\% confidence interval: 0.943-0.992), two assessment teams were formed (each with two raters: K.W., V.P., M.K., and T.E.). The assessors were blinded to all patients' clinical and demographic data, including age, sex, clinical symptoms, and therapy.

For the analysis, MRI scans were divided into two groups containing the same number of baseline and follow-up scans. Baseline and follow-up scans from the same patient were not assigned to the same group, so that each rater team analyzed only the baseline or the follow-up scan of individual patients.

For cross-validation of the semi-quantitative WML diameter measurements, a modified 'WML change scale' was used [13] for a randomly selected subgroup of 25 patients. Here, the observers directly compared the baseline and follow-up scans of individual patients to visually estimate the change in WML (fig. 1).

\section{Statistics}

Descriptive statistics are shown as mean and SD. Normalized WML measures are reported. Group comparisons of categorical data were performed using chi-square tests. Group differences in normally distributed continuous variables (Kolmogorov-Smirnov $\mathrm{Z}$ test $\mathrm{p}>0.05$ ) were conducted using t tests. Group comparisons of non-parametric, related data were calculated using the Wilcoxon signed-ranks test, and group comparisons of non-parametric, unrelated data were calculated using Mann-Whitney $U$ tests (MWU). Correlations were analyzed using Spearman rank correlation coefficients, and for regression analyses, standard regression coefficients (beta weights) were used. The retrospectively estimated sample size for a desired power of 0.8 was 47 for each sample separately. The level of statistical significance was two-tailed ( $\alpha=$ 0.05). Statistics were computed using the SPSS software package version 18.0 (SPSS, 2009 version).

\section{Results}

Overall, MRI scans were available from 70 of the 82 (85\%) randomized patients. Missing scans resulted from patients refusing the optional MRI or from contraindica-

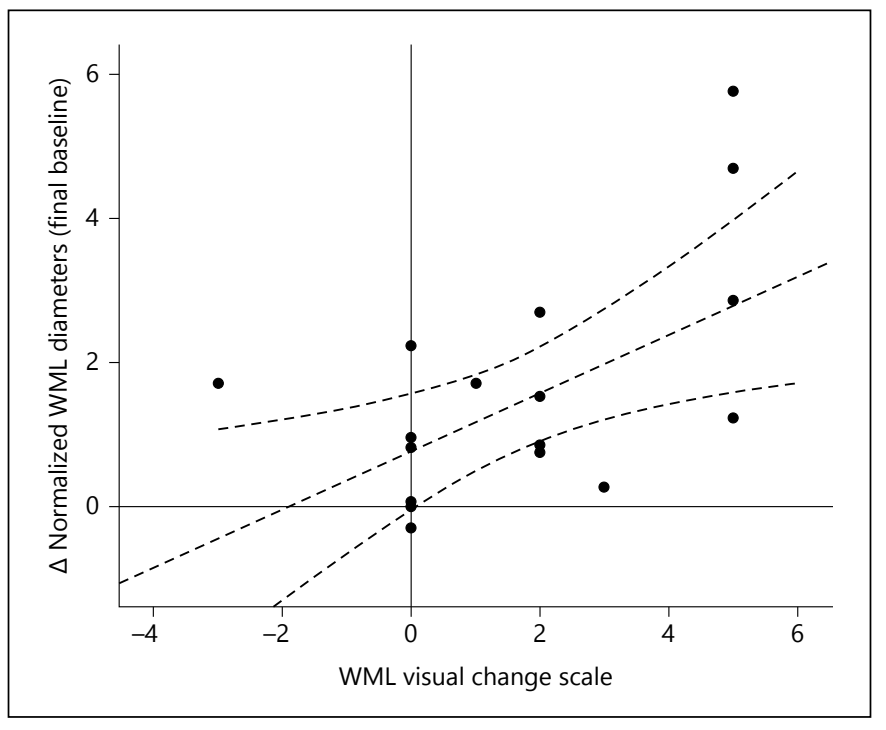

Fig. 1. Agreement of two different visual white matter lesion (WML) rating scales. Good agreement was found between the WML change scale* and the delta of WML diameter measurements normalized by the skull diameters, which were obtained at baseline and follow-up by the two independent and blinded rater teams. Both measures correlated significantly $(\mathrm{r}=0.58, \mathrm{p}=0.0039)$. * WML change scale: a sum score based on visually-classified longitudinal changes of WML of five different regions (anterior, media, posterior territory, periventricular) as increased $(+1)$, unchanged (0), or decreased (-1), modified after Prins et al. [13].

tions to MRI. After reviewing the image quality, longitudinal scans of 51 patients remained eligible for analysis. Based on consensus decision of the raters, scans from 19 male patients were excluded due to severe motion artifacts. Images from 10 further patients were excluded because their follow-up scans were performed less than 12 months after the baseline scan. Thus, scans from $41 \mathrm{pa}-$ tients were included in the final analysis ( 25 patients in the ERT group, 16 in the placebo group; mean age $43.9 \pm 9.7$ years [range 20-68 years]) with no significant differences at baseline in age, WML burden, or treatment duration. Except for one patient, all were smokers. These 41 patients had been recruited and scanned at 18 different academic centers. Patient characteristics and clinical data at baseline are listed in table 1; mean follow-up was 27 months (range 12-33 months).

\section{Comparison of WML Burden in ERT and Placebo Groups}

Overall, there were no significant differences in WML burden between the ERT and placebo groups at baseline or at follow-up (table 2). 
Table 1. Baseline patient characteristics

\begin{tabular}{|c|c|c|c|}
\hline & ERT & Placebo & $\begin{array}{l}\mathrm{p} \\
\text { value* }\end{array}$ \\
\hline $\mathrm{N}$ (women) & $25(2)$ & $16(1)$ & n.s. ${ }^{\dagger}$ \\
\hline Age, years & $45.4 \pm 9.4$ & $41.6 \pm 10.0$ & n.s. ${ }^{\ddagger}$ \\
\hline Treatment duration, days & $787 \pm 161$ & n.a. & n.s. ${ }^{\ddagger}$ \\
\hline MRI scan interval, days & $787 \pm 156$ & $785 \pm 212$ & n.s. ${ }^{\ddagger}$ \\
\hline LVPW 7 mm & $14.4 \pm 2.7$ & $13.7 \pm 2.3$ & n.s. ${ }^{\ddagger}$ \\
\hline Previous stroke ${ }^{\pi}$ & $1(4.0)$ & $1(6.3)$ & n.s. ${ }^{\dagger}$ \\
\hline Previous TIAs ${ }^{\pi}$ & 0 & 0 & n.s. \\
\hline Neuropathic pain & $14(56.0)$ & $10(62.5)$ & n.s. ${ }^{\dagger}$ \\
\hline Angiokeratoma & $15(60.0)$ & $13(81.3)$ & n.s. ${ }^{\dagger}$ \\
\hline Arterial hypertension & $8(32.0)$ & $5(31.3)$ & n.s. ${ }^{\dagger}$ \\
\hline Diabetes mellitus & 0 & 0 & n.s. ${ }^{\dagger}$ \\
\hline Hyperlipoproteinemia & $5(20.0)$ & $1(6.3)$ & n.s. ${ }^{\dagger}$ \\
\hline Atrial fibrillation & $1(4.0)$ & $1(6.3)$ & n.s. ${ }^{\dagger}$ \\
\hline eGFR & $54.7 \pm 15.4$ & $51.2 \pm 17.8$ & n.s..$^{\ddagger}$ \\
\hline Serum creatinine & $1.6 \pm 0.5$ & $1.7 \pm 0.5$ & n.s. ${ }^{\ddagger}$ \\
\hline
\end{tabular}

Values are mean \pm SD or $\mathrm{n}(\%)$. eGFR $=$ Estimated glomerular filtration rate; ERT = enzyme replacement therapy with agalsidase beta $(1 \mathrm{mg} / \mathrm{kg}$ biweekly); LVPW = end-diastolic thickness of the left-ventricular posterior wall, determined using standard 2D targeted M-mode echocardiogram recordings; n.a. = not applicable; n.s. = not significant; TIA = transient ischemic attack.

${ }^{*} \mathrm{p}<0.05$ considered significant. ${ }^{\dagger}$ Chi-square test. ${ }^{\ddagger} \mathrm{t}$ test. $\S$ Percentages calculated using the total number of the respective study group as denominator. Tा Three months or longer before inclusion.

WML burden tended to increase in both the ERT group (MWU: $\mathrm{p}=0.069$ ) and the placebo group (MWU: $\mathrm{p}=0.084)$. In the ERT group, the proportion of patients with a stable or decreased WML load was significantly higher $(44 \%, 8$ of 18$)$ than in the placebo group (31\%, 4 of 13; chi-square: $\mathrm{p}=0.014)$. The number needed to treat was 8 ; thus, 8 patients would have to be treated over the study duration of 27 months to prevent an increase in WML in one case. Although the effect size of WML load differences was strong (Cohen's $\mathrm{d}=0.58$ ), the differences between follow-up and baseline for the ERT and placebo groups were not significant.

\section{Comparison of WML Burden in the Full Study Group}

A pooled analysis of data from treated and placebo groups included in this study showed that the correlation of WML diameters $(\mathrm{mm})$ with the normalized WML measurements was highly significant at baseline $(\mathrm{r}=0.998, \mathrm{p}<0.0005)$ as well as at follow-up $(\mathrm{r}=0.997$,
Table 2. Changes in white matter lesion (WML) burden during the study follow-up period

\begin{tabular}{|c|c|c|c|}
\hline $\begin{array}{l}\text { Mean normalized WML } \\
\text { diameter }\end{array}$ & \multicolumn{2}{|c|}{ Comparator group } & $\begin{array}{l}\mathrm{p} \\
\text { value }\end{array}$ \\
\hline \multicolumn{4}{|l|}{ (A) Total study group $(\mathrm{n}=41)$} \\
\hline Baseline & $3.2 \pm 4.5$ & & $0.010^{*}$ \\
\hline \multirow[t]{2}{*}{ Follow-up } & $4.1 \pm 5.1$ & & \\
\hline & $\operatorname{ERT}(\mathrm{n}=25)$ & Placebo $(\mathrm{n}=16)$ & \\
\hline \multicolumn{4}{|c|}{ (B) Comparison of total treatment groups } \\
\hline Baseline & $3.5 \pm 5.2$ & $2.8 \pm 3.0$ & $0.843^{\dagger}$ \\
\hline Follow-up & $4.2 \pm 5.6$ & $3.9 \pm 4.3$ & $0.781^{\dagger}$ \\
\hline \multirow[t]{2}{*}{ Follow-up, minus baseline } & $0.7 \pm 1.9$ & $1.0 \pm 2.1$ & $0.741^{\dagger}$ \\
\hline & $\operatorname{ERT}(\mathrm{n}=18)$ & Placebo $(\mathrm{n}=13)$ & \\
\hline \multicolumn{4}{|c|}{ (C) Comparison of treatment and placebo patients $\leq 50$ years } \\
\hline Baseline & $2.54 \pm 4.48$ & $2.45 \pm 2.84$ & $0.737^{\dagger}$ \\
\hline Follow-up & $2.8 \pm 4.1$ & $3.8 \pm 4.3$ & $0.373^{\dagger}$ \\
\hline Follow-up, minus baseline & $0.3 \pm 1.7$ & $1.4 \pm 2.1$ & $0.125^{\dagger}$ \\
\hline
\end{tabular}

ERT $=$ Enzyme replacement therapy with agalsidase beta $(1 \mathrm{mg} / \mathrm{kg}$ biweekly); normalized WML diameter = normalized by the individual anterior-posterior skull diameter. ${ }^{*}$ MWU test. ${ }^{\dagger}$ Wilcoxon test. In (A), increases in WML burden were observed in the overall study group. In (B), no significant WML burden differences were found between the entire ERT and placebo groups at baseline, at follow-up, or over time. In (C), in contrast, the younger ERT and placebo patients ( $\leq 50$ years; $\mathrm{n}=31$ ) had similar WML burdens at baseline, but at follow-up the ERT group's WML burden increased $0.3 \pm 1.7$, whereas the WML burden increased by $1.4 \pm 2.1$ in placebo patients (Wilcoxon test: $\mathrm{p}=0.026$; see fig. 3 ).

$\mathrm{p}<0.0005)$. At baseline, the overall WML burden correlated with age $(\mathrm{r}=0.524, \mathrm{p}<0.0005$; fig. 2$)$. The baseline WML burden $(0.11 \pm 0.14)$ in patients up to 35 years of age ( $n=6$; age $28.0 \pm 6.2$ years) was lower than in older patients (WML burden: $3.8 \pm 4.6$; $\mathrm{n}=35$; age $46.7 \pm 7.3$ years; MWU: $\mathrm{p}<0.0005)$. Twenty-six percent ( 9 of 35 ) of patients over 35 years of age (mean age: $43.1 \pm 4.2$ years; 1 woman) also had a relatively low WML burden $(\mathrm{p}=0.41)$, defined as the mean total WML diameter $<2$ SDs above the mean of the younger patients (fig. 2).

Baseline WML burden correlated significantly with follow-up WML burden $(\mathrm{r}=0.866, \mathrm{p}<0.0005)$. WMLs were more often found in the periventricular region $(41 \%$ of WMLs), with $28 \%$ in the anterior territory, $21 \%$ in the middle, and $11 \%$ in the posterior territory.

LVPW thickness correlated with WML burden at baseline and at follow-up ( $\mathrm{r}=0.527$ and 0.526 , respectively; $\mathrm{p}<0.0005)$, whereas baseline serum creatinine and 


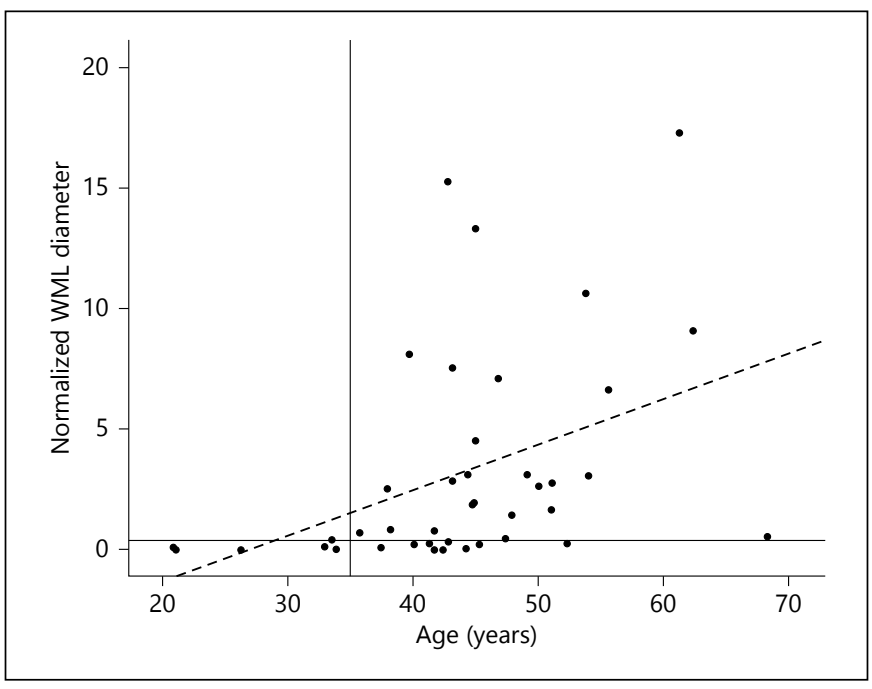

Fig. 2. Baseline white matter lesion (WML) burden of the entire study group $(\mathrm{n}=41)$. Lesion-diameter measurements were normalized using the sagittal anterior-posterior skull diameter for each individual as an internal standard. Normalized WML diameter $=$ normalized by the individual anterior-posterior skull diameters. Baseline WML burden correlated significantly with age in the FD cohort $(\mathrm{n}=41, \mathrm{r}=0.564 ; \mathrm{p}<0.0005)$. Patients up to 35 years of age ( $n=6$; left quadrant) showed only slight WML compared with older patients. Nine of $35(26 \%)$ patients above 35 years of age had a low WML burden (lower-right quadrant), which means $<2$ $\mathrm{SD}$ above the mean of the younger patients (lower-left quadrant).

eGFR did not $(\mathrm{r}=0.147, \mathrm{p}=0.357$ and $-0.206, \mathrm{p}=0.176$, respectively). Patients who had renal events, defined as a $33 \%$ increase in eGFR during the trial $(\mathrm{n}=12)$, did not have more WMLs at baseline or follow-up than patients without renal events $(n=29)$.

\section{Comparison of WML Burden in ERT and Placebo} Groups by Patient Age

Although there were no significant differences in the mean age of the patients in the ERT and placebo groups (table 1), the age distribution of patients over 50 years of age differed between groups, as seven of the 10 patients over 50 years old were in the ERT group. These older patients had a significantly higher WML burden at follow-up $(9.0 \pm 7.1$ vs. $3.1 \pm 4.0$; MWU: $\mathrm{p}=0.03$ ), as well as significantly higher increases in WML burden over time compared with patients aged $\leq 50(2.2 \pm 1.5$ vs. $0.6 \pm 1.9 ;$ MWU: $p=0.011)$.

Due to the disproportionate distribution of older patients, a subgroup analysis was performed, excluding patients $>50$ years of age. The remaining 18 ERT-treated patients did not differ from the patients in the placebo group $\leq 50$ years old $(n=13)$ in age (mean $41.1 \pm 6.5$ years

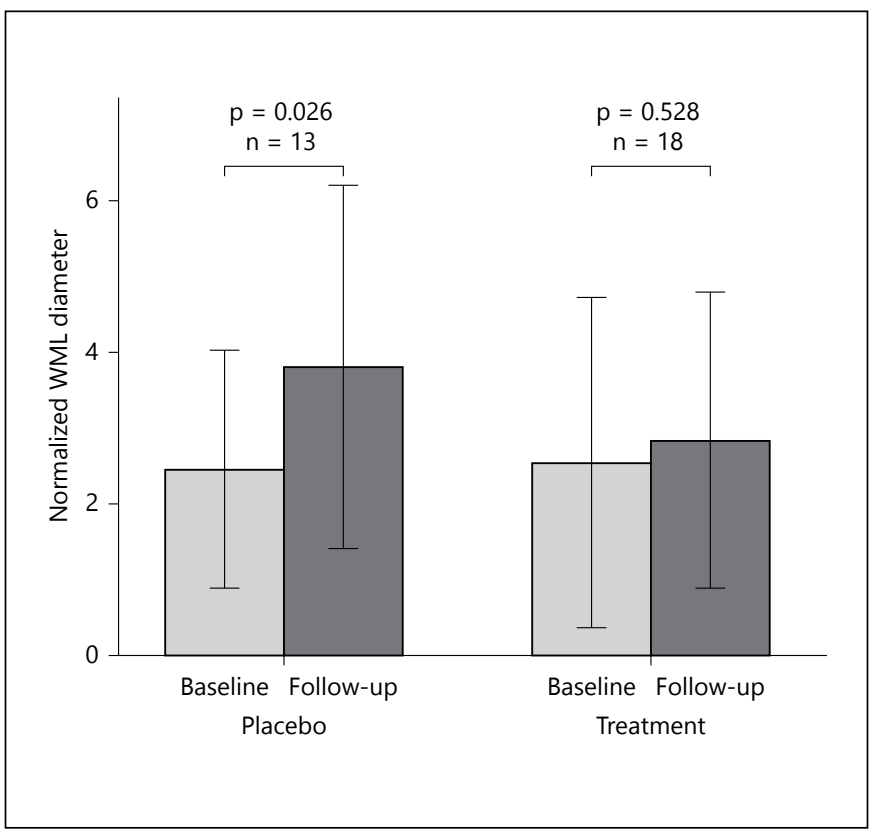

Fig. 3. White matter lesion (WML) changes in ERT and placebo patients $\leq 50$ years old. Bars show the mean overall WML burden measured as the total lesion diameter $(\mathrm{mm})$. Error bars indicate \pm 2SE. WML patients under ERT did not increase over the mean 27-month observation period (Wilcoxon test: $\mathrm{p}=0.528$ ), whereas the WML load increased significantly in the placebo group (Wilcoxon test: $\mathrm{p}=0.026$ ). vs. $38.6 \pm 8.1$ years; $\mathrm{t}$ test: $\mathrm{p}=0.341)$, treatment duration (812 \pm 169 days vs. $824 \pm 187$ days; t test: $\mathrm{p}=0.850)$, follow-up time ( $806 \pm 159$ days vs. $837 \pm 187$ days; $\mathrm{t}$ test: $\mathrm{p}=$ 0.622 ), vascular risk factors, or WML burden at either baseline or follow-up (MWU: baseline, $\mathrm{p}=0.737$; followup, $\mathrm{p}=0.373$ ). The WML burden of the younger patients in the ERT group was relatively stable during the trial, while that of the younger patients in the placebo group increased during the trial (Wilcoxon test: $\mathrm{p}=0.026$; fig. 3 ).

The WML burden at baseline and follow-up in the younger subgroup also correlated positively with LVPW thickness (baseline, $\mathrm{r}=0.611, \mathrm{p}<0.0005$; follow-up, $\mathrm{r}=$ $0.593, p=0.001)$. When controlled for age, the association of LVPW thickness and WML burden remained significant (partial correlation: LVPW-WML at baseline $\mathrm{r}=$ $0.374, \mathrm{p}=0.046$; LVPW-WML at follow-up $\mathrm{r}=0.407, \mathrm{p}=$ 0.028). Using analysis of covariance to control for age, patients with higher LVPW thickness (median split: mean LVPW thickness $15.6 \pm 1.7 \mathrm{~mm}$, range 13.5-18.4 mm) had an increased WML burden compared with patients with a lower LVPW thickness $(11.7 \pm 1 \mathrm{~mm}$, range 9.4$13.4 ; \mathrm{F}=29.2, \mathrm{p}<0.0005)$. Linear regression analysis, with 
normalized total WML diameter at follow-up as a dependent variable, and age, LVPW, and eGFR as independent variables, yielded only baseline LVPW as a significant predictor of WMLs $(\beta=0.468, \mathrm{p}=0.32)$.

Among the younger patients, renal events were observed in 10 patients and were equally distributed between the study groups: $20 \%$ (5 of 25) in the ERT group and $31 \%$ (5 of 16) in the placebo group (chi-square: $p=$ 0.701). Patients with an increase in creatinine from a baseline of $33 \%$ or more did not have more WMLs (MWU: $\mathrm{p}=0.546)$. There was no correlation between WML burden and eGFR $(\mathrm{r}=-0.133, \mathrm{p}=0.476)$ or serum creatinine $(\mathrm{r}=0.108, \mathrm{p}=0.562)$.

\section{Comparison of Stroke Incidence in the ERT and Placebo Groups}

At baseline, clinical brain infarction had been reported in one patient in each of the ERT and placebo groups. However, baseline MRIs showed signs of brain infarction in $20 \%$ ( 5 of 25 ) of patients in the ERT group and $19 \%$ (3 of 16) of patients in the placebo group. At follow-up, MRIs showed that $13 \%$ ( 2 of 16 ) of patients in the placebo group and 4\% (1 of 25) of patients in the ERT group had developed new infarcts.

With regard to classification and location, six of the baseline strokes (five in the ERT and one in the placebo group), and all three follow-up strokes, were silent. Three patients had territorial strokes, five had lacunar strokes, one had a watershed stroke, and two had multiple strokes. Four strokes were located within the posterior circulation territory, four within the middle cerebral artery territory, one within the border zone between the middle and posterior cerebral artery circulation, and two strokes had multiple locations.

Patients with baseline or follow-up strokes $(\mathrm{n}=11)$ tended to be older $(48.6 \pm 8.2$ vs. $42.2 \pm 9.8$ years; t test: $\mathrm{p}=0.063$ ), have more WMLs at baseline (MWU: $\mathrm{p}=$ 0.023 ) and follow-up (MWU: $\mathrm{p}=0.043$ ), and have a significantly greater increase in WML burden (MWU: $\mathrm{p}=$ 0.005) during the study compared with patients without stroke. Patients with stroke showed an increase in LVPW thickness $(15.5 \pm 2.7$ vs. $13.6 \pm 2.3 \mathrm{~mm}$; MWU: $\mathrm{p}=0.044$ ), but no differences in vascular risk factors compared with patients without stroke were seen during the study.

\section{Pulvinar Sign}

At baseline, five men (22\%) in the ERT group and four men $(27 \%)$ in the placebo group showed an increased signal in the lateral posterior thalamus ('pulvinar sign') on
T1-weighted images that did not change over the observation period. Patients with and without the pulvinar sign did not differ in mean age or WML burden at baseline. Similarly, patients with the pulvinar sign did not have more strokes than those without the pulvinar sign at baseline (chi-square: $\mathrm{p}=0.245$ ).

\section{Discussion}

This study analyzed the efficacy of ERT with agalsidase beta in reducing the expected progression of WMLs and stroke risk in patients with FD and mild-to-moderate renal impairment. The overall data demonstrated a significant mean increase in the WML burden over 27 months. As shown in previous cross-sectional studies, which found the WML burden to be significantly increased at a mean age of about 40 years compared with age-matched healthy controls $[12,14]$, WMLs were not apparent in patients before the age of 35 and tended to increase in size with age in our study. Of particular note, we report the first evidence of clinically significant stabilization of, or reduction in, WML load in patients treated with agalsidase-beta ERT. These findings, in this subset of 31 advanced renal patients, only further emphasize the importance of early treatment of men in childhood, as recommended by published guidelines for this Xlinked disease that causes symptoms in young children $[15,16]$.

In our analysis, WMLs were most pronounced within the periventricular regions, which is in accordance with previous reports [17]. Accumulation of Gb3 in vascular endothelial and smooth muscle in classical FD, leading to WML development, may be particularly pronounced in the long perforating arteries [17]. From an autopsy study of a patient with FD who died after 2.5 years of ERT therapy, we can speculate as to how agalsidase beta may slow the progression of WMLs [18]. In this case, the cerebrovascular endothelial cells were found to be free from Gb3. The absence of endothelial Gb3 due to agalsidase-beta treatment might delay the progression of WMLs caused by reduced cerebral microangiopathy. In turn, a reduction in the WML progression rate could be a marker of reduced risk of stroke or cognitive decline in FD [6]. The significance of cerebrovascular involvement in FD is highlighted by the fact that, in our sample, $22.5 \%$ of patients had had a prior or recent stroke, of which five were silent. This is above the expected background rate of stroke for the same age group in the general population [19]. Two patients un- 
der placebo and one patient under ERT developed new silent strokes during the study. However, due to the small sample size and the relatively short observation period, the possibility of a reduction in stroke frequency related to agalsidase-beta ERT treatment cannot be confirmed by our results.

Our study population was restricted to patients with FD who also had kidney impairment, which usually occurs in older classically affected men [1]. An association between kidney disease, cognitive impairment, and deep WML has been reported independently of FD in the general population [20,21], although we did not find any association between renal function and WML burden in this study. We found a significant association between age and vascular risk factors only for LVPW thickness and younger age among those patients both with and without significant WMLs at baseline.

Several studies have reported an association between left-ventricular hypertrophy and WML burden in middle-aged patients with arterial hypertension [22, 23]. Although the mechanism has not been elucidated, these data suggest that the association of cardiac and cerebral injury could be due to a generalized impact of arterial hypertension. However, the patients in our study did not have hypertension [3]. Moreover, Barbey et al. have shown that left-ventricular hypertrophy and intima-media thickness of the carotid artery are independent of blood pressure in FD [24]. They suggest that the cardiac chondrocyte Gb3 deposits have little impact on cardiac pathology but that the plasma of patients with FD may contain a growth-promoting factor that triggers both cardiomyocyte proliferation and vascular smooth muscle cell hypertrophy [24].

\section{Limitations}

This study has a number of limitations. There is no consensus on the optimal measure of WML burden; while a number of visual scales are available that are easy to perform, supervised semi-automated methods can provide more information on location and size, as well as continuous data, but are time-consuming [25-27]. Lesion counting was considered an alternative method; however, given the potential for confluent lesions to be identified in longitudinal analyses, we considered this method might have led to an underestimation of overall lesion burden and subsequent progression over time. We used a 3-mm slice thickness for the FLAIR-weighted images and a 5-mm gap for the axial T1 images, and thus may have missed smaller lesions. Image-to-image variation may also have been introduced by using the sagittal anterior-posterior diameter for the scans. As only hardcopy scans (films) of the MRIs were available, standard procedures for digital WML measurement allowing comparisons with commonly employed methods could not be applied, and we did not distinguish between WML and leucoaraiosis. Although the method we used in this study was a visuo-manual assessment, reliability analyses were undertaken to ensure consistency between raters. The small group size, restricted to patients with FD and kidney impairment, and the high variability of the lesion burden in the patients mean that our results may not be applicable to the wider population of patients with FD.

\section{Conclusions}

In summary, although limited to a small number of patients, this analysis provides the first evidence that agalsidase beta is an effective ERT for reducing the WML burden in patients with FD aged 50 years or younger. This cohort, with mild-to-moderate renal impairment, already had a significant WML burden and high inter-individual variability that was not associated with the degree of kidney dysfunction but was associated with the degree of leftventricular hypertrophy. These advanced patients with increased LVPW thickness and stroke may have exhibited a higher cerebrovascular risk. These findings should be replicated in a larger sample to confirm the efficacy of agalsidase beta therapy in decreasing WML progression and the presumed consequence of reducing the risk of stroke in patients with FD.

Importantly, however, even in patients with advanced renal disease, those who had less severe disease manifestations had the best outcomes. This indicates that ERT may be beneficial when initiated in childhood or adolescence in classically affected males with FD, to optimally prevent the vascular ischemia and occlusive pathology that leads to WMLs and strokes.

\section{Ethical Statement, Specific Contributions, and Conflict of Interest Statement}

The data reported in this manuscript have not been previously published and are not under consideration elsewhere. We stated within the methods section that institutional review boards at all sites have approved the protocol and that all patients gave written informed consent. 
All authors have agreed to the conditions noted on the Authorship Agreement Form (ClinicalTrials.gov Identifier: NCT 00074984). Institutional review boards at all sites approved the protocol. All patients gave written informed consent. A.F. has access to patient consent forms from any patient, and we have them on file in case they are requested by the editor.

\section{Specific Contributions to the Manuscript}

A.F.: study concept and design, analysis and interpretation, writing. M.G.: analysis and interpretation. K.W.: analysis and interpretation. A.S.: statistics, critical revision of the manuscript for important intellectual content. R.J.D.: critical revision of the manuscript for important intellectual content. K.S.: critical revision of the manuscript for important intellectual content.
The authors wish to thank the four MRI assessors who performed the data collection for this study: Kerstin Wildberger, Valeska Pape, Max Kinateder, Pia Eitner.

\section{Disclosure Statement}

The ISS was sponsored by Genzyme-Sanofi. A.F. received unrestricted grants and speaker's honoraria from Genzyme-Sanofi and Shire HGT. M.G., K.W. and A.S. report no disclosures. R.J.D. is a consultant for Genzyme Corp, Synageva BioPharma, and GSK, has stock or stock options in Amicus Therapeutics and Synageva BioPharma, has grant support from Genzyme Corp, and receives royalties from Genzyme-Sanofi and Shire HGT. K.S. receives support for ISS research from Genzyme-Sanofi and educational program support from Genzyme-Sanofi, Amicus, and Shire HGT.

\section{References}

1 Desnick RJ, Ioannou YA, Eng CM, Valle D (eds): $a$-Galactosidase A deficiency: Fabry disease; in Scriver CR, Beaudet AL, Sly WS (eds): The Metabolic and Molecular Bases of Inherited Disease, ed 8. New York, McGrawHill, 2001, pp 3733-3774.

-2 Eng CM, Banikazemi M, Gordon RE, Goldman M, Phelps R, Kim L, Gass A, Winston J, Dikman S, Fallon JT, Brodie S, Stacy CB, Mehta D, Parsons R, Norton K, O'Callaghan M, Desnick RJ: A phase $1 / 2$ clinical trial of enzyme replacement in Fabry disease: pharmacokinetic, substrate clearance, and safety studies. Am J Hum Genet 2001;68: 711-722.

3 Banikazemi M, Bultas J, Waldek S, Wilcox WR, Whitley CB, McDonald M, Finkel R, Packman S, Bichet DG, Warnock DG, Desnick RJ: Agalsidase-beta therapy for advanced Fabry disease: a randomized trial. Ann Intern Med 2007;146:77-86.

$\checkmark 4$ Fellgiebel A, Müller MJ, Ginsberg L: CNS manifestations of Fabry's disease. Lancet Neurol 2006;5:791-795.

5 Fellgiebel A, Müller MJ, Mazanek M, Baron K, Beck M, Stoeter P: White matter lesion severity in male and female patients with Fabry disease. Neurology 2005;65:600-602.

6 Debette S, Markus HS: The clinical importance of white matter hyperintensities on brain magnetic resonance imaging: systematic review and meta-analysis. BMJ 2010; 341:c3666.

7 Barkhof F, Scheltens P: Imaging of white matter lesions. Cerebrovasc Dis 2002;13(suppl 2):21-30.

8 Venketasubramanian N, Röther J, Bhatt DL, Pasquet B, Mas JL, Alberts MJ, Hill MD, Aichner F, Steg PG; REACH Investigators: Twoyear vascular event rates in patients with symptomatic cerebrovascular disease: the REACH registry. Cerebrovasc Dis 2011;32: 254-260.
-9 Moore DF, Scott LT, Gladwin MT, Altarescu G, Kaneski C, Suzuki K, Pease-Fye M, Ferri R, Brady RO, Herscovitch P, Schiffmann R: regional cerebral hyperperfusion and nitric oxide pathway dysregulation in Fabry disease: reversal by enzyme replacement therapy. Circulation 2001;104:1506-1512.

10 Moore DF, Altarescu G, Ling GS, Jeffries N, Frei KP, Weibel T, Charria-Ortiz G, Ferri R, Arai AE, Brady RO, Schiffmann R: Elevated cerebral blood flow velocities in Fabry disease with reversal after enzyme replacement. Stroke 2002;33:525-531.

11 Fazekas F, Chawluk JB, Alavi A, Hurtig HI, Zimmerman RA: MR signal abnormalities at $1.5 \mathrm{~T}$ in Alzheimer's dementia and normal aging. AJR Am J Roentgenol 1987;149:351-356.

-12 Fellgiebel A, Keller I, Marin D, Müller MJ, Schermuly I, Yakushev I, Albrecht J, Bellhäuser H, Kinateder M, Beck M, Stoeter P: Diagnostic utility of different MRI and MR angiography measures in Fabry disease. Neurology 2009;72:63-68.

13 Prins ND, van Straaten EC, van Dijk EJ, Simoni M, van Schijndel RA, Vrooman HA, Koudstaal PJ, Scheltens P, Breteler MM, Barkhof F: Measuring progression of cerebral white matter lesions on MRI: visual rating and volumetrics. Neurology 2004;62:1533-1539.

14 Crutchfield KE, Patronas NJ, Dambrosia JM, Frei KP, Banerjee TK, Barton NW, Schiffmann R: Quantitative analysis of cerebral vasculopathy in patients with Fabry disease. Neurology 1998;50:1746-1749.

15 Desnick RJ, Brady R, Barranger J, Collins AJ, Germain DP, Goldman M, Grabowski G, Packman S, Wilcox WR: Fabry disease, an under-recognized multisystemic disorder: expert recommendations for diagnosis, management, and enzyme replacement therapy. Ann Intern Med 2003;138:338-346.

16 Eng CM, Germain DP, Banikazemi M, Warnock DG, Wanner C, Hopkin RJ, Bultas J, Lee
P, Sims K, Brodie SE, Pastores GM, Strotmann JM, Wilcox WR: Fabry disease: guidelines for the evaluation and management of multi-organ system involvement. Genet Med 2006;8:539-548.

17 Albrecht J, Dellani PR, Müller MJ, Schermuly I, Beck M, Stoeter P, Gerhard A, Fellgiebel A: Voxel based analyses of diffusion tensor imaging in Fabry disease. J Neurol Neurosurg Psychiatry 2007;78:964-969.

18 Schiffmann R, Rapkiewicz A, Abu-Asab M, Ries M, Askari H, Tsokos M, Quezado M: Pathological findings in a patient with Fabry disease who died after 2.5 years of enzyme replacement. Virchows Arch 2006;448:337-343.

19 Zhou XP, Woodford-Richens K, Lehtonen R, Kurose K, Aldred M, Hampel H, Launonen V, Virta S, Pilarski R, Salovaara R, Bodmer WF, Conrad BA, Dunlop M, Hodgson SV, Iwama T, Järvinen H, Kellokumpu I, Kim JC, Leggett B, Markie D, Mecklin JP, Neale K, Phillips R, Piris J, Rozen P, Houlston RS, Aaltonen LA, Tomlinson IP, Eng C: Germline mutations in BMPR1A/ALK3 cause a subset of cases of juvenile polyposis syndrome and of Cowden and Bannayan-Riley-Ruvalcaba syndromes. Am J Hum Genet 2001;69:704-711.

20 Kurella Tamura M, Wadley V, Yaffe K, McClure LA, Howard G, Go R, Allman RM, Warnock DG, McClellan W: Kidney function and cognitive impairment in US adults: the Reasons for Geographic and Racial Differences in Stroke (REGARDS) study. Am J Kidney Dis 2008;52:227-234.

21 Kuriyama N, Mizuno T, Ohshima Y, Yamada K, Ozaki E, Shigeta M, Mitani S, Kondo M, Matsumoto S, Takeda K, Nakagawa M, Watanabe Y: Intracranial deep white matter lesions (DWLs) are associated with chronic kidney disease (CKD) and cognitive impairment: a 5-year follow-up magnetic resonance imaging (MRI) study. Arch Gerontol Geriatr 2013; 56:55-60. 
22 Sierra C, de la Sierra A, Paré JC, Gómez-Angelats E, Coca A: Correlation between silent cerebral white matter lesions and left ventricular mass and geometry in essential hypertension. Am J Hypertens 2002;15:507-512.

23 Selvetella G, Notte A, Maffei A, Calistri V, Scamardella V, Frati G, Trimarco B, Colonnese C, Lembo G: Left ventricular hypertrophy is associated with asymptomatic cerebral damage in hypertensive patients. Stroke 2003; 34:1766-1770.
24 Barbey F, Brakch N, Linhart A, RosenblattVelin N, Jeanrenaud X, Qanadli S, Steinmann B, Burnier M, Palecek T, Bultas J, Hayoz D: Cardiac and vascular hypertrophy in Fabry disease: evidence for a new mechanism independent of blood pressure and glycosphingolipid deposition. Arterioscler Thromb Vasc Biol 2006;26:839-844.

25 Scheltens P, Erkinjunti T, Leys D, Wahlund LO, Inzitari D, del Ser T, Pasquier F, Barkhof F, Mäntylä R, Bowler J, Wallin A, Ghika J, Fazekas F, Pantoni L: White matter changes on CT and MRI: an overview of visual rating scales. European Task Force on Age-Related White Matter Changes. Eur Neurol 1998;39: 80-89.
26 Anbeek P, Vincken KL, van Osch MJ, Bisschops RH, van der Grond J: Probabilistic segmentation of white matter lesions in MR imaging. Neuroimage 2004;21:1037-1044.

27 Jack CR Jr, O’Brien PC, Rettman DW, Shiung MM, Xu Y, Muthupillai R, Manduca A, Avula R, Erickson BJ: FLAIR histogram segmentation for measurement of leukoaraiosis volume. J Magn Reson Imaging 2001;14:668676. 